\title{
THE ROLE OF ELECTRIC FIELDS AND ION CONCENTRATIONS IN THE FORMATION AND STABILIZATION OF HIGH-n RYDBERG STATES
}

\author{
A. HELD and E. W. SCHLAG* \\ TU München, Institut für Physikalische Chemie, Lichtenbergstr. 4, 85748 \\ Garching bei München, Germany
}

(Received 22 December 1997)

ZEro Kinetic Energy (ZEKE) spectroscopy relies on electrons produced through delayed field ionization of the narrow band of high- $n$ Rydberg states which exist just below the ionization limit of each ionic eigenstate. Using the unique properties of these weakly bound, stable, high- $n$ Rydberg states (ZEKE states) below the ionization limit rather than the unbound states above the limit, as in PES, leads to an improvement in resolution of more than two orders of magnitude. Several different types of ZEKE experiments, each designed to probe the formation and stability of these states, are presented here. These experiments were performed with pulsed and static electric fields of different magnitude and duration at different ion concentrations. The results indicate an enhanced ZEKE state decay with increasing electric field strengths and an enhanced formation and stabilization with increasing ion concentrations. A strong interplay between field strength and ion concentration ZEKE state formation is demonstrated. The strong influence of electric fields and ion concentrations on the physical properties of the ZEKE state, above and below the classical ionization threshold, is also demonstrated through late time (tens of microseconds) decay rate measurements.

Keywords: ZEKE photoelectron spectroscopy; Rydberg states; laser ionization; field ionization

\section{INTRODUCTION}

Rydberg states are formed through the excitation of an electron into an orbital which is best described by atomic orbitals belonging to

\footnotetext{
${ }^{*}$ Corresponding author.
} 
shells well outside the valence shells of the atom(s). The bound electronic Rydberg levels become closer together as the energy is increased and eventually merge into a ionization continuum. Although the discovery of these states is now over 100 years old [1], their often non-intuitive physical properties are still the subject of intense study today. Nowhere is this more prevalent than in the relatively young field of ZEro Kinetic Energy (ZEKE) spectroscopy [2]. ZEKE spectroscopy is a high resolution photoelectron spectroscopy which relies on electrons/ions produced through delayed pulsed field ionization of the narrow band of extremely stable high- $n$ Rydberg states existing just below the ionization limit of each rotational, vibrational and electronic ionic eigenstate. The long lifetimes of these states, first observed by Reiser, et al. [3] in 1988, were unanticipated since they deviate strongly from what one would expect based on the decay rates extrapolated from low- $n$ Rydberg states and on the assumed rapid rate of autoionization and/or predissociation. Interactions which cause Rydberg states to decay through autoionization and/or predissociation can only take place near the ionic core; since the probability of finding a Rydberg electron near the core scales as $1 /$ $n^{3}$, interactions that lead to autoionization or predissociation scale as $n^{-3}$ and the lifetimes as $n^{3}$. The high- $n$ Rydberg states detected in ZEKE experiments have much longer lifetimes which scale as $\sim n^{5}$ ! The orbitals describing the long-lived high- $n$ Rydberg state must therefore be significantly modified in order to avoid core interactions. The production of these stable states has been studied by several groups [4-12] and is now concluded to be strongly dependent on the physical modifications produced by electric fields and ion concentrations present during the formation process. In this article we summarize some of the most recent findings focusing on experiments performed at the Technical University in Munich. Although several experiments whose results corroberate well with those determined in Munich are discussed, this account is not intended to be a review of ZEKE spectroscopy.

Central to the understanding of the experimentally determined importance of electric field and ion-charge effects on the physical properties of Rydberg states is a basic knowledge of the ZEKE spectroscopic technique. ZEKE spectroscopy is defined by an electron emanating from an excited specially stabilized high- $n$ Rydberg state, a 
ZEKE state. The distance of this electron from the atomic or molecular core is increased for these special states such that the interactions between the two are strongly reduced. The core finds itself in an ionic eigenstate, the electron acting solely as a slowly orbiting spectator. This electron, once separated from the molecular core, is on the threshold of escaping the high Rydberg orbit with zero energy remaining. The selection of these states from completely ionized states depends on the fact that, despite their respective distances, the ionic core and the orbiting electron remain together as a neutral species. The ZEKE technique hinges on the fact that atoms and molecules in these special high Rydberg states live for a very long time and that every ion state has its own Rydberg series, hence its own ZEKE states. Discrimination against lower lying, short-lived Rydberg states is achieved simply by waiting some time after laser excitation, during which time a small electric field is used to separate the «background》 ions from the targeted long lived Rydberg neutrals.

Figure 1 shows a set of Rydberg series and the ionization continua associated with the vibrational and rotational eigenstates of an arbitrary ion. As illustrated in this figure, the fate of the initially excited Rydberg state is multifold. Excitation into a Rydberg state built on an eigenstate above the ionization threshold can result in decay by coupling directly to the continuum or by couplings mediated through isoenergenic Rydberg states of different intermediate levels which can eventually lead to an open ionization or predissociation channel. Since the total energy of the system must always be conserved, these decay channels are governed by electron-ion energy exchange and thus restricted by selection or propensity rules.

The Rydberg states of high principle quantum number $n$, measured in typical ZEKE experiments, can be described as having strongly mixed orbital angular momentum, $\ell$, with very low weight of the lowest $\ell$ components. The centrifugal barrier prevents an electron of given angular momentum $\ell$ from approaching the core closer than a distance of $r \sim \ell(\ell+1) / 2$. This modification of the Rydberg orbital provides a vanishing probability of finding the ZEKE electron near the core and is the commonly accepted element of most models explaining the stability of these states. The stabilized high- $n$ Rydberg series hugging each eigenstate represent such $\ell$-mixed states and can be thought of as «islands of stability» lying immune to the detrimental 


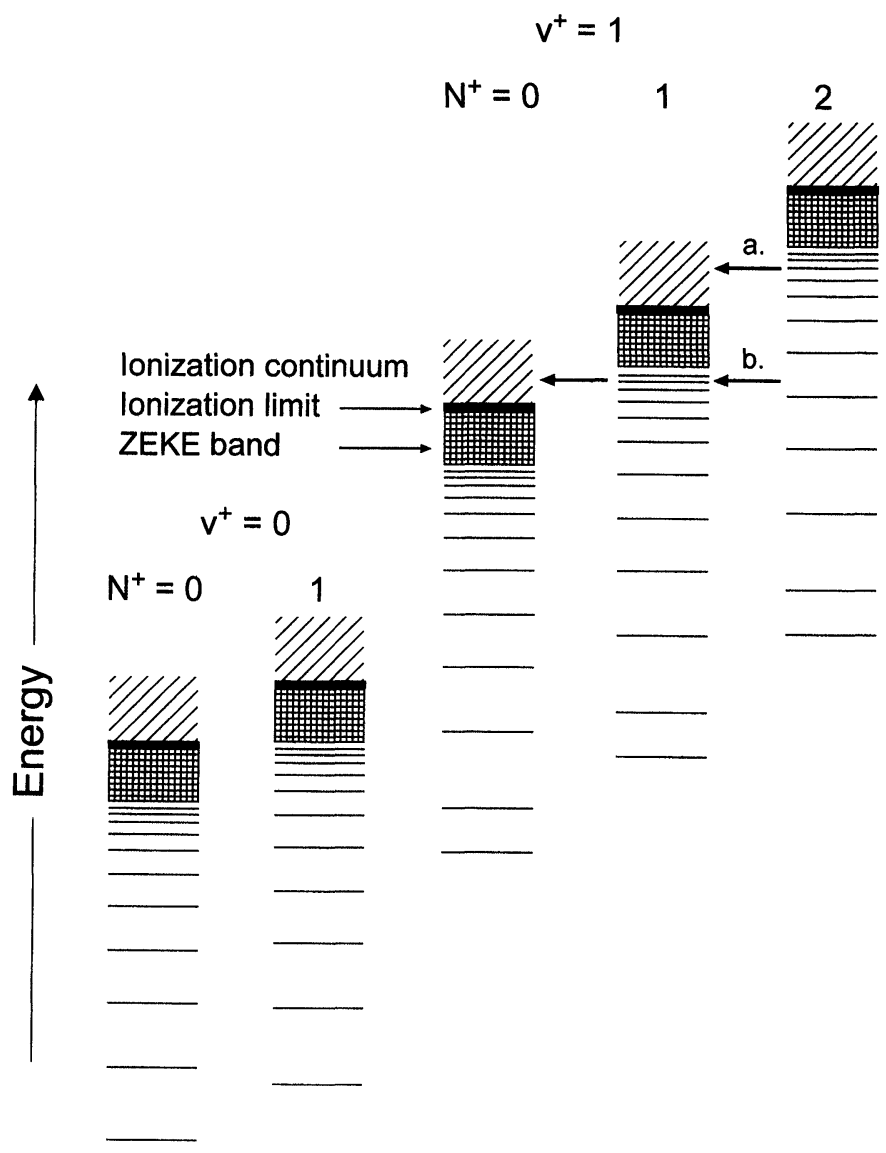

FIGURE 1 Schematic eigenstate spectrum of a molecule with bound Rydberg series and ionization continua associated with each ionization limit. Horizontal transitions illustrate (a) direct autoionization and (b) autoionization coupled through an intermediate level (channel coupling). Autoionization and predissociation/internal conversion occur as a second step, which follows the initial excitation of an atom or molecule into a state above the IP and produces a de-excitation from this state to give a positive ion with lower energy and an ejected electron (autoionization) or two lower energy fragments (predissociation).

effects of the ionization continuum and/or quasi-continuum of states. These islands of Rydberg state stability have been recently found to exist as high as $20 \mathrm{eV}$ above the IP! [13]

An electric (or magnetic) field is requisite for the ion-neutral separation process in the ZEKE technique, however, in addition to 
this practical application, the electric field is now realized to have a more subtle and profound consequence. In 1993 Chupka [14] outlined a proposal suggesting that the long lifetimes of ZEKE states have their origins at least in part as a result of Stark mixing of different orbital angular momentum states ( $\ell$ states) induced by electric fields. Electric fields induce Stark splittings in the $n$-Rydberg manifold, which for a given $n$ leads to new, nondegenerate Stark eigenstates. The presence of a dc field breaks the spherical symmetry of the Coulombic field and thus $\ell$ is no longer a good quantum number. The eigenstates in the presence of an electric field are Stark states, characterized by quantum numbers $n, k$ (defined by parabolic quantum numbers $n_{1}$ and $n_{2}$, which can take on values form 0 to $n-1)$ and $m$ [15]. When these Stark states are described in terms of field free eigenstates characterized by $n, \ell$ and $m_{\ell}$ one finds that a large range of $n$ values contributes to a given single Stark state. Recall that, electrons in orbitals that avoid interactions with the ionic core, that is, orbitals with high- $\ell$ character, have slower decay rates compared to those that can penetrate into the ionic core, orbitals with low- $\ell$ character. It follows then that when angular momentum state coupling occurs ( $\ell$-mixing), in the presence of a small electric field, the field free low- $\ell$ lifetimes are greatly enhanced by mixing with the longer living high- $\ell$ states. Whatever the coupling between low $\ell$ and high- $\ell$ states we must remember that these processes are subject to microscopic reversibility (i.e., low- $\ell$ states $\leftrightarrow$ high- $\ell$ states). Hence, any mechanism that enables the production of high- $\ell$ states from low- $\ell$ states also favors the production of low- $\ell$ states from high- $\ell$ states. Further dilution of the low- $\ell$ content of the eigenstates can be realized when the Rydberg neutrals experience the electric field of nearby ions, here in addition to the spherical symmetry also the cylindrical symmetry of the homogeneous electric field is broken. Under these conditions the projection of the orbital angular momentum on an axis is no longer fixed; i.e., $m$ is not a good quantum number. Mixing between optically accessible low- $m$ states and «dark» higher- $m$ Stark states, which have zero low- $\ell$ content, greatly enhances dilution of the decay rates due to the $(n-|k|)$-fold $m_{\ell}$ degeneracy of the Stark levels.

Recently it has been discovered that the electric field used to separate direct ions from neutrals and which induces Stark mixing can also produce a detrimental enhancement of the decay rates at higher 
field strengths [5, 6, 16-19]. In several systems, higher field strengths have been shown to couple lower- $\ell$ components into the description of the eigenstates by inducing deeper Stark state mixing [7, 20, 21]. Electric field induced Stark mixing can also relax the restrictive selection rules between the electron and the ion core and thus allow the energy exchange required for processes leading to open decay channels which may be closed without an electric field [22-25]. In addition, the field can produce a second order Stark splitting effect which lifts the degeneracy of different $m$ levels to such an extent that the initially populated $m$ levels cannot mix in with other levels thus restricting the preferentially large high- $\ell$ phase space and allowing microscopic reversibility back into low- $\ell$ states [6]. Notwithstanding, the addition of high ion concentration at the point of excitation has been found to suppress these field effects through rapid stabilization of the Rydberg states [4, 6, 8-12]. The compensating effect of ion density over the field induced depletion of stable Rydberg state formation occurs with surprisingly few ions and scales with the increasing field strength $[9$, 10]. The influence and interplay between these two environmental perturbations on the ZEKE spectra of benzene will be demonstrated here.

\section{EXPERIMENTAL CONDITIONS}

A schematic of the ion optics system, used in the Munich experiments [26] to separate direct ions from neutral Rydbergs, is shown in Figure 2. Although the apparatus is specifically designed to separate cations from the neutrals of relatively large molecular species, the principles illustrated in Figure 2 are the basis of all ZEKE experiments. The neutral molecular beam (here benzene) enters the region between the first two sets of ion optic plates where either a static or delayed pulsed electric field is applied. This allows for laser excitation in the presence or absence of an electric field. Excitation occurs through a two color, two photon process, the first laser excites the molecules into an electronic intermediate state and the second laser is used to scan through the Rydberg manifold. Ions can be produced directly, in the excitation region, by two photon, one color absorptions from the first 

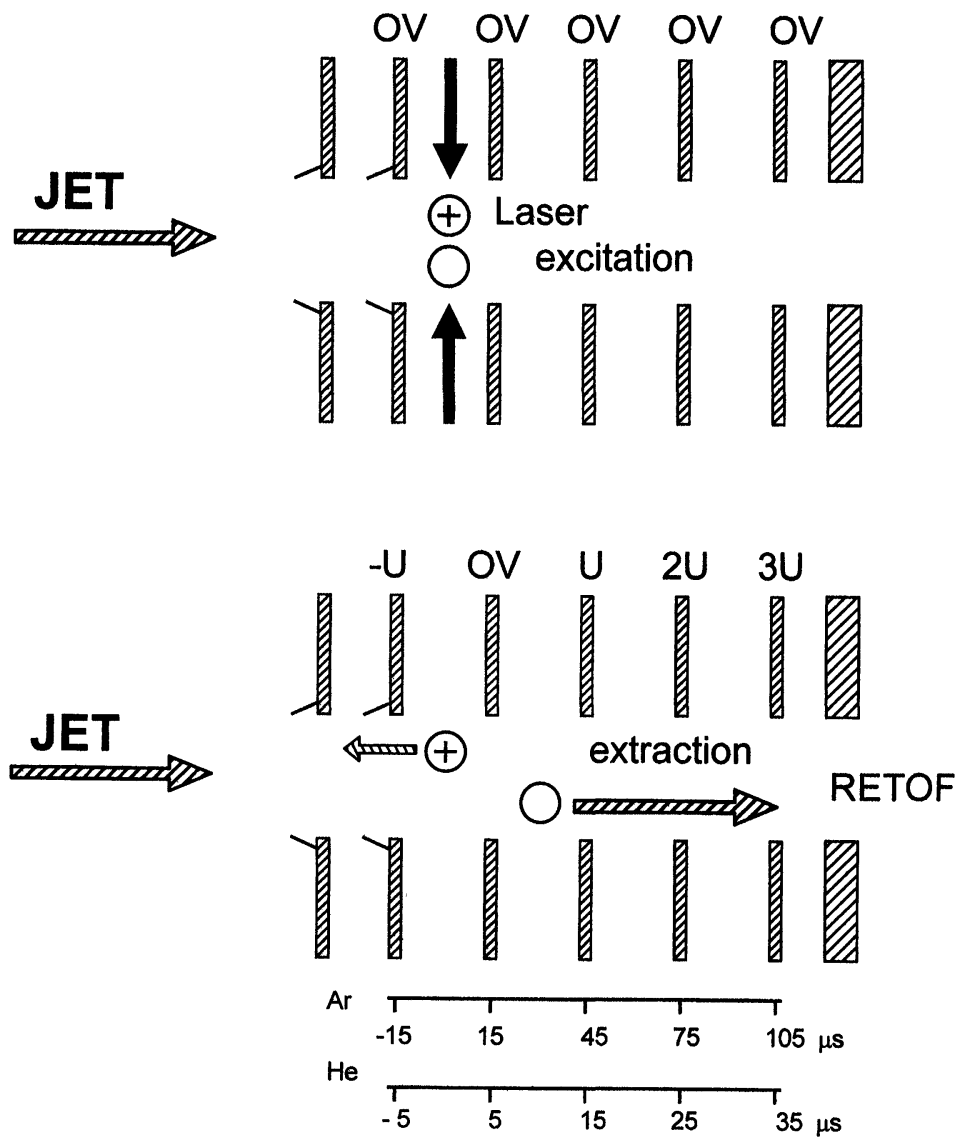

FIGURE 2 Schematic of the experimental arrangement: A supersonic molecular beam crosses the two counterpropagating lasers in the region between plates P1 and P2 (top). The excitation either occurs under field free conditions (when both plates are at ground potential) or in the presence of a separating field of 0.2 to $3 \mathrm{~V} / \mathrm{cm}$ (when P2 is at ground potential and $\mathbf{P} 1$ is at $-\mathrm{U}$ ). In the former case the separating field is applied $10 \mathrm{~ns}$ to $1 \mathrm{us}$ after laser excitation. The separating field, whether static or delayed, retards the direct ions with respect to the Rydberg (ZEKE neutrals) molecules (bottom). To insure proper separation and signal, the field is applied through the ion optics up to the point of extraction. The molecules are ionized by a $200 \mathrm{~V} / \mathrm{cm}$ field applied to the last two sets of plates. This pulsed delayed field accelerates the now ionized ZEKE molecules towards the detector. The very bottom part of the figure shows the jet flight time through the ion optics where $\mathrm{He}$ or Ar are used as a carrier gas.

laser, which has an energy greater than one half the ionization energy of benzene. Ions are also produced through the autoionization processes depicted in Figure 1. 
The separation of the background ions from the long-lived Rydberg neutrals begins between the first two plates when the field is applied. The field retards the ions while the neutrals pass through the ion optic plates at the speed of the carrier gas. The time scales, shown below the ion optic plates in Figure 2, illustrate the flight time from laser excitation to pulsed field extraction, approximately $24 \mu$ s for $\mathrm{He}$ and $66 \mu \mathrm{s}$ for Ar. These times are fixed by a variable, fast rising positive extraction pulse which is applied to the last two sets of extraction plates producing an electric field of $200 \mathrm{~V} / \mathrm{cm}$. This field ionizes the neutrals which are then extracted into a reflectron time of flight (RETOF) mass spectrometer. The observed ion signal arises solely from the field ionized Rydberg states which are stable enough to survive the relatively long drift time through the ion optics i.e., those states which exist in the «islands of stability».

\section{FIELD EFFECTS}

We described above the many possible decay channels which can open as a result of increased fields being present before the stabilization of the optically accessible Rydberg states can occur. The resulting, dramatic loss in signal is demonstrated in Figure 3. Here, two spectra of benzene were recorded under identical conditions except in one case the molecules were excited in the presence of a $1 \mathrm{~V} / \mathrm{cm}$ field and in the other case the same field was delayed $100 \mathrm{~ns}$ after laser excitation i.e., the molecules were excited under near field-free conditions. The shape of the spectra are, in general, asymmetric, having a sharp rising high frequency edge and a long trailing low frequency edge. The high frequency portion of the spectra has been empirically shown [10] to correspond to transitions from selective intermediate state levels to high- $n$ Rydberg states built on the $0^{0+}$ ion state. The lower frequency portion of the spectra corresponds to the lower- $n$ Rydberg states converging on these $0^{0+}$ rotational levels. A series of such spectra were recorded at field strengths from 200 to $300 \mathrm{mV} / \mathrm{cm}$. The results of these experiments show that the dramatic, relative loss in signal, when the molecules are excited in the presence of an electric field increases with the increasing magnitude of the field. In fact, at the highest fields, the in-field signal disappears completely. Figure 4 shows the same field 


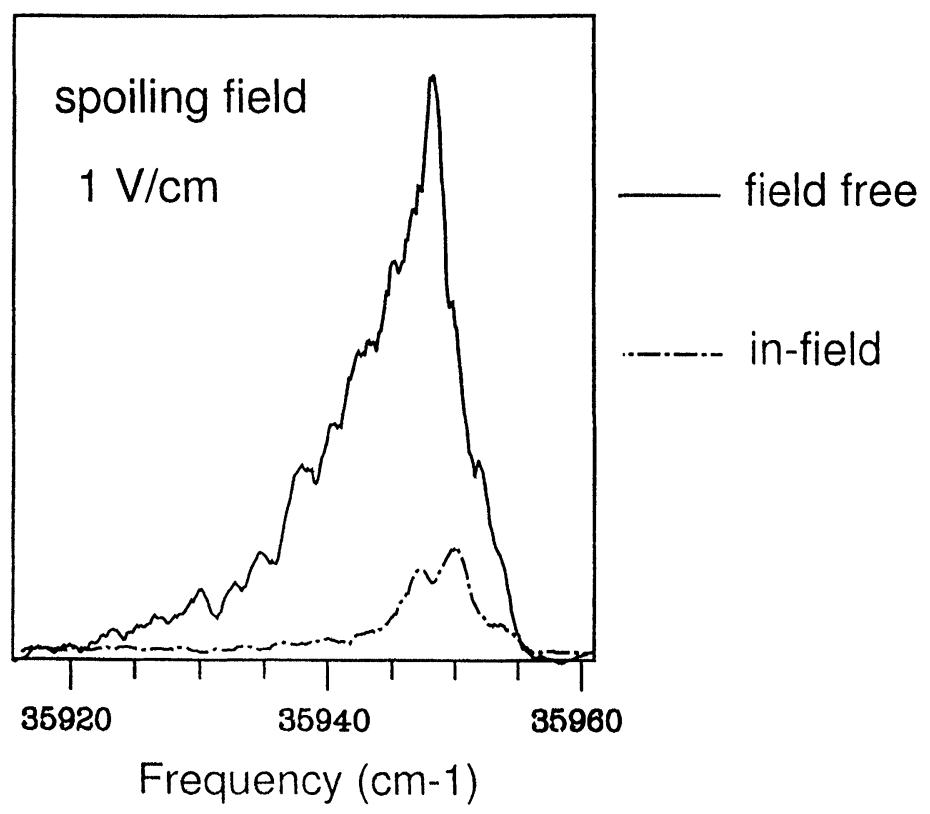

FIGURE 3 Effect of static and delayed dc electric fields on the pulsed field ionization (PFI) spectra near the $0^{0+}$ transition of benzene via the $\mathrm{S}_{1} 6^{1} J=1 \mathrm{~K}=1$ intermediate state. The dashed spectrum was recorded where a dc field of $1 \mathrm{~V} / \mathrm{cm}$ was present during laser excitation. The solid spectrum was recorded where the same $1 \mathrm{~V} / \mathrm{cm}$ field was applied $100 \mathrm{~ns}$ after laser excitation.

free (top) and in-field (bottom) spectra as shown in Figure 3, but here their intensities have been scaled. Simulated spectra [27], calculated for transitions from the $K=1 J=1$ intermediate state to the allowed ionic rotational states are superimposed on the high frequency edges of both spectra. The overlay of the simulated ionic transitions onto the experimental spectra (obtained under field free and in-field excitation conditions) clearly illustrates that, in addition to the overall loss in signal, when excitation occurs in-field, there is also a relatively stronger erosion of the lower energy edge of the spectrum (compared to that where excitation takes place under field free/delayed field conditions). As depicted in Figure 1, the lower energy edge of each ZEKE band corresponds to the lower- $n$ Rydberg levels converging to several different rotation levels (Fig. 1). The stronger lower energy edge degradation of the in-field spectra is therefore not surprising 

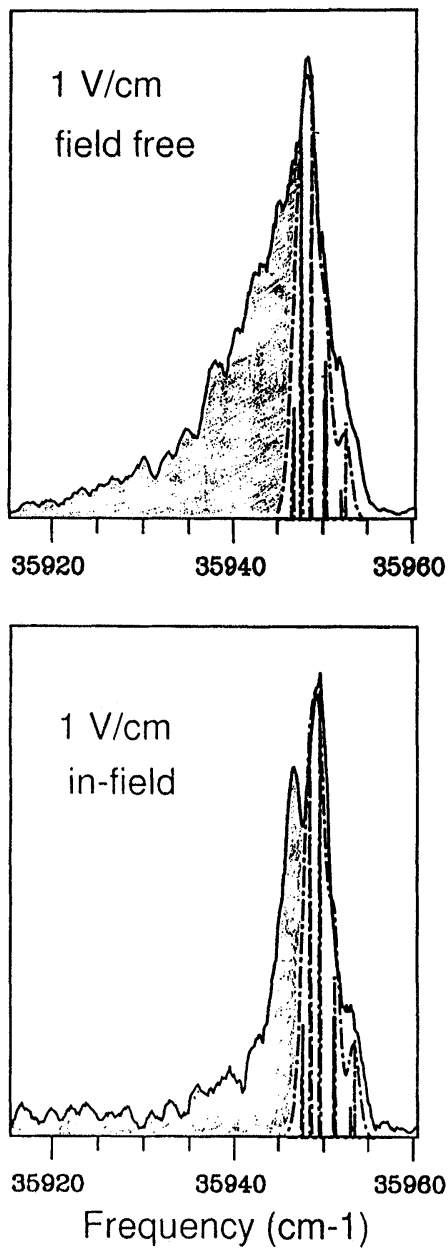

FIGURE 4 Effect of electric field on the widths of the PFI spectra. The top panel shows the PFI spectra near the $0^{0+}$ transition of benzene via the $S_{1} 6^{1} J=1 K=1$ intermediate state where the $1 \mathrm{~V} / \mathrm{cm}$ separating field is applied $100 \mathrm{~ns}$ after photoexcitation. The bottom panel shows the PFI spectrum recorded under the same conditions except the field was present during excitation. The intensities in both panels are normalized. The simulated spectra (dashed) show the relative positions of the rotational states calculated from rotational constants determined by Lindner et al., Ref [27].

considering that the corresponding lower- $n$ states are already at the lower boundary of the «island of stability»; hence they are particularly sensitive to even small additional field-induced destabilization. 
The presence of an electric field does not change the total crosssection of the optical absorption into the Rydberg states. Excitation in the presence of an electric field must therefore provide a parallel process for the dissappearance of the optical Rydberg states in lieu of conversion into ZEKE states which then live long enough to be ionized by a delayed pulsed field and be detected. In particular, an intentionally applied electric field may reduce the longer lifetimes in the distribution of lifetimes describing the optically accessible Stark Rydberg states. The loss of the longer living, stabilizing, contributions to the overall description of the Stark mixed Rydberg state can arise through one or more decay mechanisms described earlier. In a polyatomic system, such as benzene, it is likely that a number of decay processes are operative, the weight of each depends on the applied field strength.

\section{ION EFFECTS}

The experiments on benzene presented thus far were carried out under conditions where the ion concentration in the laser excitation region is kept very low. This is accomplished by reducing the intensity of the intermediate state laser which can produce ions through direct onecolor two photon ionization. The experiments carried out with $1 \mathrm{~V} / \mathrm{cm}$ spoiling field, applied during and after laser excitation, at low densities were repeated at higher ion density. The ion density was increased by increasing the intensity of the intermediate state laser beam. Spectra obtained at high ion densities under in-field and field free excitation conditions are shown in Figure 5. Comparing the spectra recorded at these higher ion densities with those at low ion density (see Figs. 3 and 4), we see that for the in-field experiments, the losses, due to field enhanced decay can be restored by increasing the ion concentration. When the in-field spectrum is normalized (see bottom of Fig. 5) to the intensity of the delayed field spectrum we see that the spectral widths are nearly identical. These results indicate that, at least for the typical condition employed here, the addition of ions at the point of excitation, where laser excitation occurs in the presence of an electric field, enhances the formation of ZEKE states. This result is less clear for field free/delayed field experiments since there the field does not 

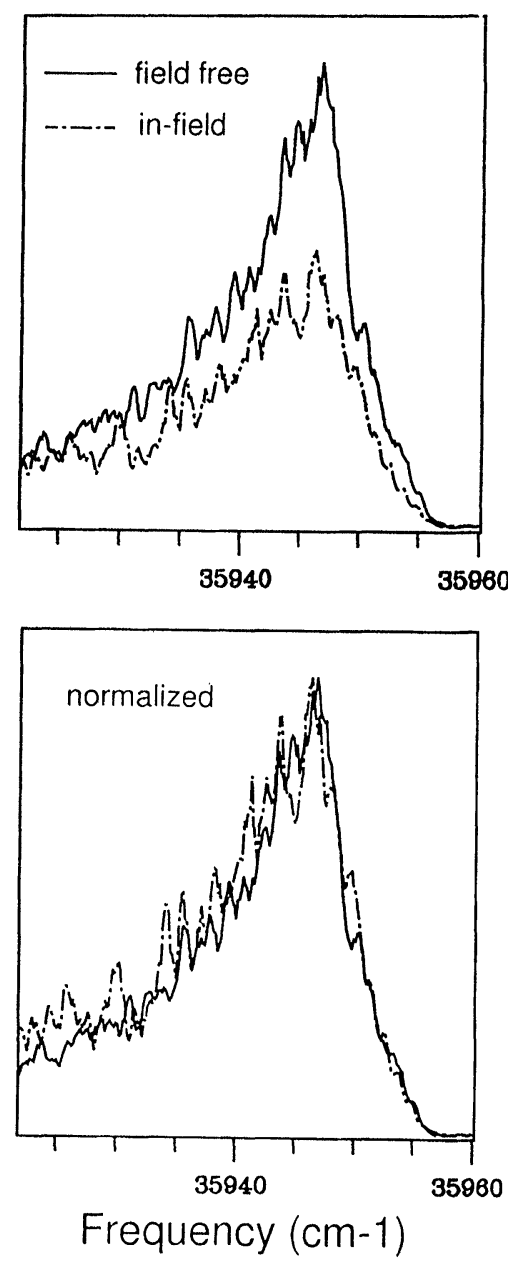

FIGURE 5 Effect of ion concentration on the pulsed field ionization spectra near the $0^{0+}$ transition of benzene via the $\mathrm{S}_{1} 6^{1} J=1 K=1$ intermediate state. The dashed spectra were recorded where a dc field of $1 \mathrm{~V} / \mathrm{cm}$ was present during laser excitation. The solid spectra were recorded where the same $1 \mathrm{~V} / \mathrm{cm}$ field was applied $100 \mathrm{~ns}$ after laser excitation. The spectra shown in the bottom panel are the same as those in the top panel but their intensities are normalized.

dramatically effect the ZEKE intensity. Also, as the intermediate state laser intensity is increased, both the in-field and delayed field signals increase due to the increased population of the $S_{1}$ intermediate state of benzene. 
In an attempt to decouple increases in signal due to ion induced enhancement of the ZEKE formation process from increases in signal simply due to an increase in the population in the $S_{1}$ intermediate state, a third laser was used to independently produce additional ions. The third laser comes from doubling part of the second harmonic of a YAG laser. The fixed frequency of the third laser $(\lambda=266 \mathrm{~nm})$ ionizes states from the intermediate $S_{1}$ level populated by the first laser. The laser is not resonant with any ionic eigenstate of benzene and thus produces additional ions without producing additional ZEKE states. Both the intermediate state laser and the scanning laser intensities were kept low and hence the ion concentration, without the third laser, was kept very low. The spectrum with the third laser was more intense and has a more extensive low energy edge than that recorded without the third laser, that is, without additional ions produced by the third laser. This shows that even under nearly field free conditions, where only our small experimental stray field $(40 \mathrm{mV} / \mathrm{cm})$ is present [28], ions enhance ZEKE state formation.

Lifetime enhancement of Rydberg states by surrounding ions, injected in a similar way, was also observed by Vrakking et al. [8] in recent vibrational wave packet experiments on $I_{2}$. The pump-probe scans of $I_{2}$ were significantly enhanced when additional ions were introduced using an independent laser. The average measured lifetimes of the Rydberg states, where additional ions were present, was shown to be significantly lengthened compared to that where the additional ions were absent. In a complimentary experiment, Merkt et al. [4] used high ion concentrations to reshuffle the Stark manifold in the presence of a small applied field. In this experiment the Stark electric field was decreased after the long-lived ZEKE states had already been formed to allow remixing of lower $\ell$ and $m$ states which produced a decay in signal. How far the field strength needed to be lowered to produce the reshuffling depended on the ion concentration present. This ion enhanced reshuffling (remixing) of the Stark mixed Rydberg states in $\mathrm{NO}$ is analogous to the initial ion enhanced ZEKE state formation process we observed in the benzene experiments.

The benzene experiments, described above, clearly demonstrate that the presence of an electric field $(>200 \mathrm{mV} / \mathrm{cm})$ during laser excitation hinders the production of ZEKE states and that the addition of ions enhances the formation of ZEKE states. To investigate whether there 
is a true reciprocal effect between field strength and ion concentration on ZEKE state formation a series of measurements were made at higher ion concentration with further increasing field strengths. The ion concentration was augmented by increasing the strength of the intermediate state laser until the in-field spectrum, where a $1 \mathrm{~V} / \mathrm{cm}$ separating field is applied, had the same intensity and width as that where the field is delayed. Adding additional ions, at this point has no effect on the relative spectra. The ZEKE state quantum yield can therefore be said to be $100 \%$. The field was then increased to $2 \mathrm{~V} / \mathrm{cm}$ and then to $3 \mathrm{~V} / \mathrm{cm}$. The resulting spectra show similar losses in signal strength an spectral width (losses from the low energy edge) with increasing field strengths, as those where ion concentrations were kept low. This verifies that the attrition due to external fields can be compensated for by added ions and vice versa.

The strong interplay between electric fields and ion concentrations on ZEKE state production is particularly important since these experimental parameters are very often difficult to control in most ZEKE spectrometers. An example of a seemingly contradictory result which is likely to have resulted from such an interplay is that of Alt et al. [29]. In these experiments a three laser system was used to independently produce background benzene $d_{6}$ ion while observing changes in the benzene $h_{6}$ ZEKE signal. The conclusions reached in these experiments were that the injection of additional ions had no effect on the ZEKE signal and thus no effect on ZEKE state production. In the light of the new results presented here, the experiments of Alt et al., were most probably performed under conditions where the ion density, produced through $1+1$ ionization from the laser used to excite the benzene $h_{6}$ intermediate state, was already sufficient to counter the detrimental effects of the electric field. In other words, the ZEKE state quantum yield was already $100 \%$ before the injection of additional ions. Reaching this saturation limit with relatively few ions can easily be realized when laser excitation occurs under near field-free conditions, where only small stray fields are present and the applied spoiling field is delayed. Since the stray fields can vary in different spectrometers, the critical effects of ions on the ZEKE spectra obtained in these different experimental set-ups may sometimes appear to be inconsistent. 


\section{LIFETIMES OF ZEKE STATES: BELOW THE CLASSICAL IONIZATION THRESHOLD}

In the experiments described above it is clear that the presence of ions is instrumental in the ZEKE state formation process. Ion induced $m$ mixing converts optical Rydberg states into longer lived $\ell, m$-mixed ZEKE states. This process competes with the decay of optical states. The ratio. of the two rates, $\ell, m$-mixing and decay, respectively, determines branching between two different product states formed from the same optical states. As shown here, both rates can be varied experimentally, the manifestation of which appears as the loss or gain of signal after some given delay time following laser excitation. The signal intensity is a measure of how many ZEKE states are formed or, in other words, how many states are formed with very limited direct electron-ionic core interactions. The decay rate at long times (tens of microseconds) is a measure of how limited these interactions are. To examine the long-time component of the ZEKE state decay we have applied a novel experimental approach which measures the decay rates independently of how the ZEKE states are formed [18]. Briefly, ZEKE spectra were obtained where the ZEKE neutrals were extracted at different delay times, typically $12-24 \mu$ s after laser excitation. The decay of the integrated ZEKE signal at these longer times was used as a measure of the decay rate of the ZEKE states. The series of experiments showed that the measured decay rates are independent of whether laser excitation occurs in the presence or absence of an electric field. Recall, however, that excitation in the presence of an electric field does have a profound effect on the absolute intensity of the ZEKE spectra. The shapes of the ZEKE spectra were also independent of how late the field ionization pulse was applied. It can therefore be concluded that the conditions at excitation strongly affect the formation of ZEKE states but do not affect their late time decay rates and that all states contributing to the ZEKE spectra are thoroughly mixed and decay at the same rate. These experiments demonstrate the clear distinction between early and late effects in ZEKE states. Ion concentrations, which play an important role in ZEKE state formation, were shown to have little effect on the decay rate long after excitation/formation. 
The late time decay rate was found to be strongly affected by the presence of an electric field after laser excitation through the point of delayed pulsed field ionization. When the field is turned off before pulsed field ionization, the resulting decay rate increases by a factor of 4-5. Switching the electric field on and off several times before extraction produces dramatic losses in signal which scales with the switching time. Signal losses, produced by switching the field back to zero or nearly zero has also been observed for Ar and was shown recently to be dependent on the ion concentration present [4]. The results of the field switching experiments are consistent with the $\ell$ mixing argument presented earlier. Turning off the electric field allows the superposition states containing high- $\ell$ character to remix and take up additional low- $\ell$ character. Each time the field is switched on and off the ensemble of states is reshuffled and more long-lived Stark states with high- $\ell$ character are converted to short lived Stark states with low- $\ell$ character. The result is the observed loss in ZEKE signal at longer times. One can conclude from these experiments that the ZEKE states, once formed, are very effectively decoupled from the ionic core and that by applying and sustaining a small dc field from laser excitation through pulsed field extraction the coupling can be further diminished.

\section{LIFETIMES OF ZEKE STATES: ABOVE THE CLASSICAL IONIZATION THRESHOLD}

Recently, an even more direct experimental measure of the ZEKE electron's proximity to it's ionic core was accomplished. This was done by exciting the Rydberg electron above it's classical ionization threshold and measuring how long it can live there. Above the classical ionization threshold, states which have their orbital dipole moments parallel to the applied field will promptly ionize (energy downshifted $=$ red states) while those with orbital dipoles antiparallel with respect to the field are resistant to ionization (energy upshifted states $=$ blue states). The orientations of these $\ll$ red $\gg$ and $\ll$ blue $\gg$ orbitals with respect to a homogeneous field are illustrated in Figure 6. The decay mechanisms operating on states below the classical ionization threshold are equally operative above the classical thresh- 
old, however, above the classical ionization threshold an additional autoionization channel is opened due to the coupling between blue states and the isoenergenic «red》 continua (the physics of this process is reviewed in [30]). Pictorially, one can imagine an isoenergetic reorientation of the blue orbital, shown in the right half of Figure 6, into the red orbital, shown in the left half of Figure $6(+k$ going to $-k$ ). The orbital would now have an orientation with respect to the field favorable for ionization. Note that the same net effect occurs if one fixes the orbital orientations and simply changes the field direction.

There are several reasons why the decay of the above threshold states is a particularly sensitive indicator of the ZEKE electron's proximity to the core. Above threshold ionization results from scattering off the non-hydrogenic core which couples the motions of the electron along the parabolic co-ordinates $\eta$ and $\Sigma$ [30]. The coupling is possible even if the state of the core remains unchanged after the scattering. Hence, this process is not subject to restrictive selection or propensity rules. Furthermore, the static isotropic (independent of geometry and orientation of the molecular core) part of the non Coulombic core perturbation, which is immaterial for the electron-core energy exchange, is still operative in causing the $\ll$ bluered» autoionization.

The experiment is performed as follows: the pulsed fields, illustrated below the ion optics shown Figure 7, are applied in such a way that the molecules experience a small «separating》 field of $50 \mathrm{mV} / \mathrm{cm}$ for $\sim 20 \mu$ s. After $\sim 20 \mu$ s a «spiked $\gg$ field of 1,10 and $20 \mu$ s duration is applied on top of the separating field. This field efficiently ionizes all red states but does not completely ionize the resilient blue states. Between the third and fourth plate a field is applied which has the same magnitude but opposite direction to that of the spiked field plus the separating field. This field ionizes all neutrals which survived (remained resilient, blue states) above the ionization threshold for 1 , 10 and $20 \mu$ s. The spectra shown in Figure 7 result from the detection of these ions and decreases in intensity with increasing spike field duration. The intensities were integrated to yield a decay time of $20 \pm 5 \mu$ s. The experiment was performed in the presence of relatively few ions and was repeated at higher ion density. The result was that at higher ion density there was no measurable decay of the ZEKE signal 


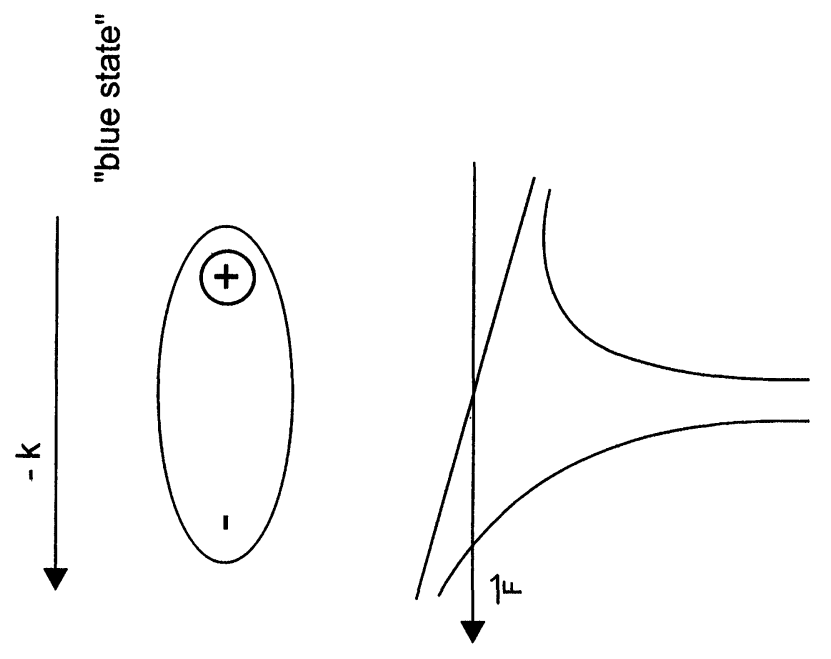

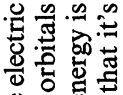
点焉焉 영 웝 氙造 렁웡 헝 눙 윰 월 오 해웅 昰志 혀ㅇㅝㅠ 돈 $\leqq$ 응

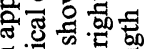
도윰월

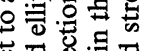

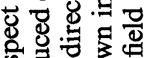

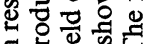

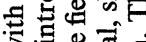
웜웜

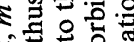
멿월 흥 훙용 สั

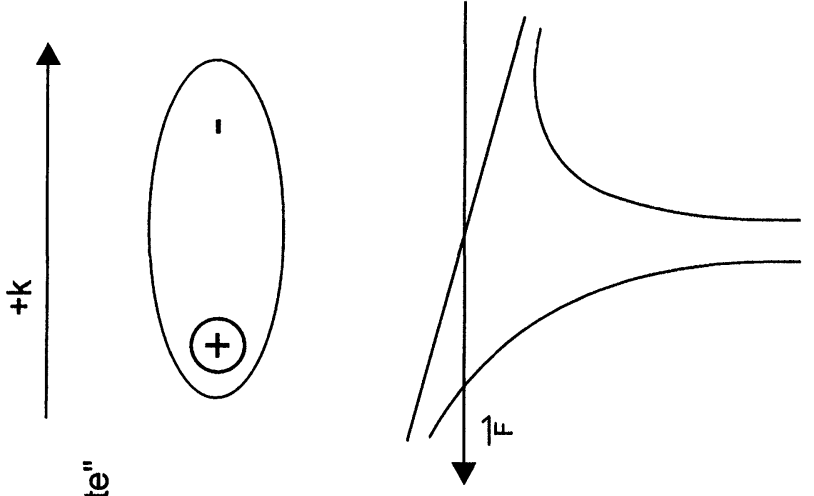
रे .

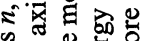
屯્ّ क 00 웡 包 운을.욜 胥造

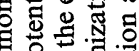
용듀 융 을 옹

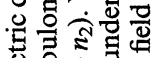
过 1 总

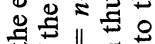
范范些总

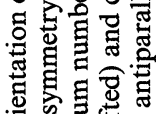

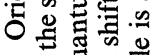
○苟灾总递

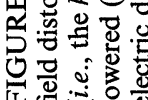




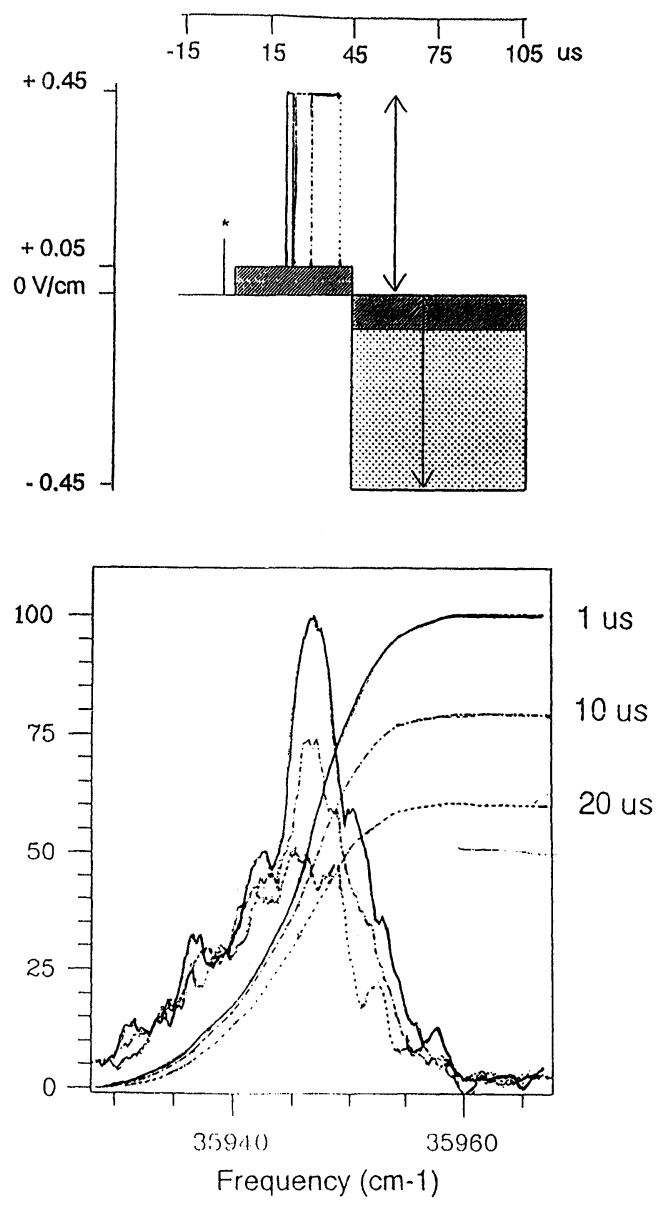

FIGURE 7 Pulsed field ionization spectra near the $0^{0+}$ transition of benzene via the $\mathrm{S}_{1}$ $6^{1} J=4 K=4$ intermediate state recorded using spike widths of $1 \mu \mathrm{s}$ (solid), $10 \mu \mathrm{s}$ (dashed) and $20 \mu$ s (dotted). The $400 \mathrm{mV} / \mathrm{cm}$ spiked fields are applied to a $50 \mathrm{mV} / \mathrm{cm}$ separating field (shaded portion of the timing scheme). The $-450 \mathrm{mV} / \mathrm{cm}$ pulse which produces the detected signal is represented by the dot filled region. The solid, dashed and dotted lines are the integrated intensities of the solid, dashed and dotted spectra recorded with 1,10 and $20 \mu$ s wide spiked pulses, respectively.

with increasing spike width. Thus the increase in ion concentration efficiently assists in the formation of ZEKE states which have orbits such that they do not return to the ion core for very, very long times. This experiment directly probes the changes in the interactions of the 
ZEKE electron with the ionic core by observing directly the change in decay rate as a function of ion concentration.

\section{CONCLUSION}

In this article we have outlined the two major environmental perturbations which effect the decay and production of highly stabilized high Rydberg states (ZEKE states). These states, which are created through electric field induced Stark state mixing, have a high degree of high- $\ell$ character which is favored by ion induced $m_{\ell^{-}}$ mixing. The high stability and thus long lifetimes of these states is an essential aspect of the field of ZEKE spectroscopy. It has been demonstrated that though small fields are necessary for Stark mixing, higher fields enhance the decay rates of the optically accessible Rydberg state resulting a loss of signal. It has also been demonstrated that higher ion concentrations, present during ZEKE state formation, counter the detrimental effects of increased fields. The reciprocity between electric field enhanced decay and ion induced stabilization of ZEKE states has also been outlined. Finally, we showed empirical evidence that high ion densities induce near complete decoupling between the ionic core and the ZEKE electron.

\section{References}

[1] Rydberg, J. R. (1890). Z. Phys. Chem., 5, 227.

[2] For recent reviews see: Schlag, E. W. and Levine, R. D. (1997). Comm. At. Mol. Phys., 33, 159; Yeretzian, Ch, Selzle, H. L. and Schlag, E. W. (1996). Eur. Mass. Spect., 2, 3; Müller-Dethlefs, K., Schlag, E. W., Grant, E. R., Wang, K. and McKoy, B. (1995). Adv. In. Chem. Phys., XC, 1; Schlag, E. W., Peatman, W. B. and Müller-Dethlefts, K. (1996). J. Electron Spectrosc. Relat. Phenom., 66, 139.

[3] Reiser, G., Habenicht, W., Müller-Dethlefs, K. and Schlag, E. W. (1988). Chem. Phys. Lett, 152, 119.

[4] Palm, H. and Merkt, F. (1997). Chem. Phys. Lett., 270, 1.

[5] Merkt, F. (1994). J. Chem. Phys., 100, 2623.

[6] Merkt, F. and Zare, R. N. (1994). J. Chem. Phys., 101, 3495.

[7] Vrakking, M.-J. and Lee, Y. T. (1995). J. Chem. Phys., 102, 8818.

[8] Vrakking, M.-J., Fischer, I., Villeneuve, D. M. and Stolow, A. (1995). J. Chem. Phys., 103, 4538.

[9] Held, A., Baranov, L. Ya., Selzle, H. L. and Schlag, E. W. (1996). Z. Naturforsch., 51a, $123 \mathrm{~b}$.

[10] Held, A., Baranov, L. Ya., Selzle, H. L. and Schlag, E. W. (1997). J. Chem. Phys., 106, 6848 . 
[11] Held, A., Baranov, L. Ya., Selzle, H. L. and Schlag, E. W. (1997). Chem. Phys. Lett., 267, 318.

[12] Martin, J. D. D., Hepburn, J. W. and Alcaraz, C. (1997). J. Phys. Chem. A., 101, 6728.

[13] Morioka, Y., Lu, Y., Matsui, T., Tanaka, T., Yoshii, H., Hayaishi, T. and Hall, R. I. (1996). J. Chem. Phys., 104, 9357.

[14] Chupka, W. A. (1993). J. Chem. Phys., 98, 4520.

[15] Stebbings, R. F. and Dunning, F. B., Rydberg States of Atoms and Molecules, (Cambridge University Press, Cambridge, 1983).

[16] Pratt, S. T. (1993). J. Chem. Phys., 98, 9241.

[17] Merkt, F., Mackenzie, S. R. and Softley, T. P. (1995). J. Chem. Phys., 103, 4509.

[18] Held, A., Selzle, H. L. and Schlag, E. W. (1996). J. Phys. Chem., 100, 15314.

[19] Dietrich, H.-J., Lindner, R. and Müller-Dethlefs, K. (1994). J. Chem. Phys., 101, 3399.

[20] Neuhauser, R. and Neusser, H. L. (1996). Chem. Phys. Lett., 253, 151.

[21] Bixon, M. and Jortner, J. (1995). J. Phys. Chem., 99, 7466.

[22] Bordas, C., Brevet, P. F., Broyer, M., Chevaleyre, J., Labastie, P. and Perrot, J. P. (1998). Phys. Rev. Lett., 60, 917.

[23] Bordas, C., Brevet, P. F., Broyer, M., Chevaleyre, J. and Labastie, P. (1987). Europhys. Lett., 3, 789.

[24] Mahon, C. R., Janik, G. R. and Gallagher, T. F. (1990). Phys. Rev. Lett., A 41, 3747.

[25] Merkt, F., Fielding, H. H. and Softley, T. P. (1993). Chem. Phys. Lett., 202, 153.

[26] Scherzer, W. G., Selzle, H. L. and Schlag, E. W. (1994). Phys. Rev. Lett., 72, 1435.

[27] Lindner, R., Sekiya, H. L., Beyl, B. and Müller-Dethlefs, K. (1993). Angew. Chem., $105,631$.

[28] Held, A., Selzle, H. L. and Schlag, E . W. (1997). J. Phys. Chem., 101a, 533.

[29] Alt, C., Scherzer, W. G., Selzle, H. L. and Schlag, E. W. (1994). Chem. Phys. Lett., $240,457$.

[30] Gallagher, T. F., Rydberg Atoms (Cambridge University Press, Cambridge 1994). 